ESJ Natural/Life/Medical Sciences

\title{
Estimation Des Concentrations Et Cartographie De La Dynamique Des Polluants Atmosperiques Particulaires Dans La Ville D’abidjan
}

\author{
N'Guessan Bi Vami Hermann, \\ Université Felix Houphouët Boigny (UFHB), Côte d'Ivoire \\ Centre Universitaire de Recherche et d'Application \\ en Télédection (CURAT), Côte d'Ivoire \\ Lazile Stephanie, \\ Université Felix Houphouët Boigny (UFHB), Côte d'Ivoire \\ Centre Universitaire de Recherche et d'Application \\ en Télédection (CURAT), Côte d'Ivoire \\ Université Felix Houphouët Boigny (UFHB), Côte d'Ivoire \\ Loukou Jerome André, \\ Université Felix Houphouët Boigny (UFHB), Côte d'Ivoire \\ Centre Universitaire de Recherche et d'Application \\ en Télédection (CURAT), Côte d'Ivoire \\ Saley Mahaman Bachir, \\ Université Felix Houphouët Boigny (UFHB), Côte d'Ivoire \\ Centre Universitaire de Recherche et d'Application \\ en Télédection (CURAT), Côte d'Ivoire \\ Kouadio Affian, \\ Université Felix Houphouët Boigny (UFHB), Côte d'Ivoire
}

Doi:10.19044/esj.2021.v17n43p116

Submitted: 24 July 2021

Accepted: 14 October 2021

Published: 31 December 2021
Copyright 2021 Author(s)

Under Creative Commons BY-NC-ND

4.0 OPEN ACCESS

Cite As:

N’Guessan Bi V.H., Lazile S., Loukou J.A., Saley M. B.,\& Affian K..,(2021). Estimation Des Concentrations Et Cartographie De La Dynamique Des Polluants Atmosperiques Particulaires Dans La Ville D’abidjan European Scientific Journal, ESJ, 17(43), 116.

https://doi.org/10.19044/esj.2021.v17n43p116

\section{Résumé}

En Côte d'Ivoire, la gestion et la sécurité sanitaire liée à la pollution atmosphérique revet un grand interêt . Dans ce même élan de gestion de la qualité de l'air l'OMS a fixé un seuil d'émission que les pays doivent intégrer 
dans leur mode de gestion. Cependant, dans les villes de grandes concentrations comme Abidjan il est parfois difficile de maitriser les paramètres d'émissions. L'objectif de cette étude est donc d'estimer les concentrations des polluants atmospreriques particulaires $\mathrm{PM}_{2.5}$ et ensuite cartographier la dynamique de ces polluants de 2015 à 2020. Pour atteindre cet objectif , les données de l'imagerie satellitaire LANDSAT ont été utilisées. Il a s'agit de calculer les réflectances de surfaces et les réflectances au sommet de l'atmosphère. Le but de ces calculs était de déduire les réflectances atmosphériques. En considérant la relation étroite existence entre les $\mathbf{P M}_{2.5}$ et la réflectance atmosphérique un modèle de régression linéaire a été conidéré pour déduire les $\mathrm{PM}_{2.5}$. Les résultats ont montré que les concentrations des $\mathrm{PM}_{2.5}$ sont de façon générale au-dessus du seuil de l'OMS. Ces valeurs sont de $31 \mu \mathrm{g} / \mathrm{m}^{3}$ en janvier 2015 , de $36 \mu \mathrm{g} / \mathrm{m}^{3}$ en janvier 2016 , de $32 \mu \mathrm{g} / \mathrm{m}^{3}$ en décembre 2016, de $33 \mu \mathrm{g} / \mathrm{m}^{3}$ en janvier 2019 et de $33 \mu \mathrm{g} / \mathrm{m}^{3}$ en janvier 2020. Cela donne une concentration moyenne de $33 \mu \mathrm{g} / \mathrm{m} 3$. Les communes de Yopougon Nord, Abobo Ouest, Cocody ouest et Port-Bouet Est sont les zones les plus pollués avec des concentrations allant de 26 à $49 \mu \mathrm{g} / \mathrm{m} 3$.

Mots clés: Abidjan, PM2.5, Particules, Pollution atmosphériques, concentrations.

\section{Estimation Des Concentrations Et Cartographie De La Dynamique Des Polluants Atmosperiques Particulaires Dans La Ville D’abidjan}

N'Guessan Bi Vami Hermann,

Université Felix Houphouët Boigny (UFHB), Côte d‘Ivoire

Centre Universitaire de Recherche et d'Application

en Télédection (CURAT), Côte d'Ivoire

\section{Lazile Stephanie,}

Université Felix Houphouët Boigny (UFHB), Côte d'Ivoire

Centre Universitaire de Recherche et d'Application

en Télédection (CURAT), Côte d'Ivoire

Université Felix Houphouët Boigny (UFHB), Côte d'Ivoire

Loukou Jerome André,

Université Felix Houphouët Boigny (UFHB), Côte d'Ivoire

Centre Universitaire de Recherche et d'Application

en Télédection (CURAT), Côte d'Ivoire

Saley Mahaman Bachir,

Université Felix Houphouët Boigny (UFHB), Côte d'Ivoire 
Centre Universitaire de Recherche et d'Application en Télédection (CURAT), Côte d'Ivoire

\title{
Kouadio Affian,
}

Université Felix Houphouët Boigny (UFHB), Côte d‘Ivoire

\begin{abstract}
In Côte d'Ivoire, management and health security related to air pollution are of great interest. In this same approach to air quality management, WHO has set an emission threshold that countries must integrate into their management method. However, in cities with a high concentration like Abidjan, it is sometimes difficult to control the emission parameters. The objective of this study is therefore to estimate the concentrations of particulate air pollutants PM2.5 and then to map the dynamics of these pollutants from 2015 to 2020. To achieve this objective, data from LANDSAT satellite imagery were used. . This involves calculating the surface reflectances and the reflectances at the top of the atmosphere. The purpose of these calculations was to deduce atmospheric reflectances. Given the close relationship between PM2.5 and atmospheric reflectance, a linear regression model was considered to derive PM2.5. The results showed that PM2.5 concentrations are generally above the WHO threshold. These values are $31 \mu \mathrm{g} / \mathrm{m} 3$ in January 2015, $36 \mu \mathrm{g}$ / m3 in January 2016, $32 \mu \mathrm{g} / \mathrm{m} 3$ in December 2016, 33 g / m3 in January 2019 and $33 \mu \mathrm{g} / \mathrm{m} 3$ in January 2020. This gives an average concentration of $33 \mu \mathrm{g} / \mathrm{m} 3$. The cities of Yopougon noth part, Abobo west part, Cocody west part and Port-Bouet east part are the most polluted areas with concentrations ranging from 26 to $49 \mu \mathrm{g} / \mathrm{m} 3$.
\end{abstract}

Keywords: Abidjan, PM2.5, Particulate Matter, Air Pollution, Atmospheric Polluants, Concentrations

\section{Introduction}

Les aérosols ont un éventail important de sources d'émissions naturelles et anthropiques, avec une dominance anthropique ayant des composés physico-chimiques s'avérant les plus nocifs. Précisons toute de même que la catégorisation des aérosols est difficilement systématisée par la classification des sources d'émissions. En effet, l'atmosphère est un espace très hétérogène constitué de plusieurs gaz et particules à des concentrations plus ou moins équilibrés pour la vie humaine.

La dispersion des polluants dépend de plusieurs paramètres dont les conditions météorologiques, climatique et la topographie locale (altitude, relief, cours d'eau, ...). Dès leur émission dans l'atmosphère, les polluants sont soumis aux phénomènes de dispersion et de transport sous l'effet des turbulences atmosphériques. Selon (Anthes, 1997 et Basly, 2000), le vent, et 
la stabilité atmosphérique sont des paramètres sérieux pour déterminer la dispersion des aérosols. Cette dispersion s'effectue essentiellement dans la couche limite atmosphérique (partie de l'atmosphère située en dessous de la troposphère libre) et dépend aussi de sa stabilité (Adon, 2019).

Les PM sont produits et éjecté dans l'air à travers les activités anthropiques, notamment par des phénomènes de combustion provenant d'une part, des secteurs résidentiels, tertiaire, du trafic routier. Et d'autre part des activités mécaniques. Il est à noter que les $\mathrm{PM}_{10}$ (particules de taille inférieure à 10 microns) et les $\mathrm{PM}_{2.5}$ (particules de taille inférieure à 2.5 microns) sont de bons indicateurs des polluants émis par le trafic (Cerema, 2014); par contre les $\mathrm{PM}_{10}$ se forment à partir des $\mathrm{PM}_{2.5}$; ils représentent en moyenne annuelle environ 60 à $70 \%$ des PM10.

. En général, les $\mathrm{PM}_{2.5}$ proviennent de la condensation des vapeurs très chaudes (Stromatas, 2013) c'est-à-dire qu'ils sont émis suite à la carbonisation de la biomasse (bois...) ; des matières organiques fossilisées (charbon, fuel, diesel). Ce sont des particules pouvant être transporter sur de grandes distances tout en subissant des mutations physico-chimiques. Elles ont une vitesse de sédimentation beaucoup plus faible que les $\mathrm{PM}_{10}$ et peuvent rester en suspension des jours voire des semaines durant (Boucher, 2012).

Les particules en absorbant ou diffusant le rayonnement solaire influent drastiquement sur les températures au sol. Elles jouent dans l'atmosphère le rôle de noyau de condensation autour desquels gravitent les molécules d'eau pour la formation des gouttelettes. Sans particules, il n'y a pas de nuages. De plus, la présence de particules dans l'atmosphère augmente la quantité du rayonnement solaire diffus au détriment du rayonnement solaire direct, ce qui influence la photosynthèse. Une fois en contact avec les aérosols le rayonnement est partiellement absorbé et/ou dissipé plus tard sous forme de chaleur. Ce mécanisme appeler aussi effet de serre a pour effet de réchauffer l'atmosphère et par ricochet réduit la densité nuageuse (Aoun, 2017).

En milieu urbain, les dépôts de particules dégradent le patrimoine immobilier en occurrence le patrimoine architectural historique, en causant altération et dégâts esthétiques qui entraînent des frais de nettoyage et d'entretiens considérables. Il est cependant difficile de chiffrer les dégâts dus aux particules étant donné que celles-ci sont généralement associées à des nuisances par d'autres polluants : SO2, pluie acide (Aguirregabiria et Mira, 2007). Les particules fines peuvent par ailleurs être absorbées par les plantes ou se déposer sur le sol. Leur degré de toxicité dépend de leur composition chimique et de leur éventuelle association à d'autres polluants. Ces particules peuvent par conséquent porter atteinte à la biosphère, directement ou via la chaîne alimentaire qui est liée aux organismes pollués.

La santé humaine est de plus en plus menacée par la forte présence des aérosols dans l'air. En effet, plusieurs études épidémiologiques ont pu 
témoigner de la nocivité des particules ; elles sont la cause de maladies respiratoires aiguë telles que les bronchites ou l'asthme chez les enfants (cachon, 2013), et aggravent l'état de santé des personnes atteinte de maladies cardiaque et pulmonaire. Ces particules sont dites contenir des composés cancérigènes. En outre, ce sont des polluants qui pénètrent facilement dans les poumons. Une exposition a plus de $15 \mu \mathrm{g} / \mathrm{m}^{3}$ pendant $24 \mathrm{~h}$ augmente la susceptibilité de leur effet sur la santé (Techarat et al., 2018).

Selon le rapport de 2016 de l'institut américain " health effects institute (hei) », la Sixieme cause de mortalité précoce dans le monde est liée à la pollution atmosphérique devant l'alcool, le manque d'activité physique et une mauvaise hygiène sanitaire. La pollution atmosphérique est responsable d'une morbidité annuelle de 4,1 millions de personnes. A ce titre, l'OMS stipule que les continents les plus touchés sont les continents africains et asiatiques avec $90 \%$ de ces décès.

Au niveau mondial, pour régulariser et restreindre les émissions et les concentrations des particules polluantes dans l'atmosphère, l'OMS a fixé des seuils de concentrations journalières et mensuelles que les pays se doivent de respecter. Les seuils pour les $\mathrm{PM}_{2.5}$ ont été plafonné à $25 \mu \mathrm{g} / \mathrm{m}^{3}$ pour les concentrations journalières et $10 \mu \mathrm{g} / \mathrm{m} 3$ pour les concentrations annuelles. Cependant depuis 2006 ; malgré cette recommandation, les niveaux ambiants de $\mathrm{PM}_{2.5}$ ne cessent de grimper. (WHO, 2016).

La télédétection est un outil et surtout un ensemble de connaissance permettant d'acquérir des informations ; soit, les caractéristiques biologiques et physiques des objets à distance et ceci sans contact matériel avec ces derniers. Ces données satellitaires sont également utilisées dans la détection des polluants dans le domaine de la qualité de l'air. On y extrait les paramètres comme le réseau routier, occupation du sol, etc. pour modéliser leurs concentrations et leurs transports.

En effet, les images fournies par capteurs passifs (c'est-à-dire dépendant de l'éclairement solaire) enregistrent les propriétés physiques des objets ; restituent les informations dans différentes longueurs d'ondes (petites et grandes longueurs d'ondes). Ces capteurs procèdent donc en mesurant l'énergie émise ou réfléchie par les objets. En outre, le rayonnement atteignant le capteur doit d'abord traverser l'atmosphère, et, bien que les capteurs soient sensibles à des longueurs d'onde peu absorbées (fenêtres atmosphériques), une variation de la quantité d'aérosols modifiera les mesures. Cette influence va dépendre de la longueur d'onde ( $\lambda$ ) (donc du capteur utilisé), du type et du diamètre des aérosols mais également de l'angle d'illumination solaire et de l'angle de visée du satellite (Horvath, 1997)).

En l'état actuel des connaissances, il convient d'améliorer les études en matière d'exposition réelle de la population à la pollution et d'acquérir une estimation précise des polluants atmosphérique dans l'espace et dans le temps. 
C’est dans ce contexte que la présente étude a été initiée. Elle porte sur la cartographie des polluants atmosphériques plus précisément sur les particules atmosphériques de tailles inférieures à $2.5 \mu \mathrm{m}\left(\mathrm{PM}_{2.5}\right)$ dans la ville d'Abidjan.

L'objectif principal de cette étude est de cette étude est de faire une analyse spatiale à l'aide des images satellitaire des polluants atmosphériques ( $\left.\mathrm{PM}_{2.5}\right)$ dans la ville d'Abidjan. De façon spécifique il s'agit de :

- faire une estimation des concentrations des polluants atmosphériques $\mathrm{PM}_{2.5}$ dans la ville d'Abidjan à partir de l'imagerie satellitaire ;

- analyser spatialement la distribution des polluants atmosphériques $\mathrm{PM}_{2.5}$ dans la ville d'Abidjan ;

- montrer les zones fortement impactées par les polluants atmosphériques $\mathrm{PM}_{2.5}$ dans la ville d'Abidjan.

\section{Analyse et traitement des données}

\section{I.1. Presentation de la zone d'etude}

La ville d'Abidjan est située la région des lagunes au sud-est de la cote d'ivoire, au bord du golfe de guinée et à une altitude de $18 \mathrm{~m}$. Localisé selon les latitudes et longitudes de $4^{\circ} 11^{\prime} 0^{\prime \prime}$ ' et $3^{\circ} 49^{\prime} 0^{\prime \prime}$ ' de longitude Ouest et $5^{\circ}$ 9' $0^{\prime \prime}$ et $5^{\circ} 31$ '0', de latitude Nord (Djossou et al., 2018), elle s'étend sur une surface de de 57735 ha soit $57 \mathrm{~km}$ sur $40 \mathrm{~km}$ soit 0,6\% et comptait en 2014, 6.351.086 habitants selon l'Institut National des Statistiques (INS)(Kouadio et al., 2019). Depuis aout 2001, Abidjan est devenue un district " district autonome d'Abidjan (Remer et al., 2005) qui regroupe quatre sous-préfectures dont Anyama, Abidjan ville, Bingerville, Brofodoumé et Songon (INS 2014). La ville d'Abidjan comprend quant à elle 11 communes dont : Yopougon, Abobo, Cocody, Adjamé, Plateau, Treichville, Koumassi, Marcory, PortBouet. Abidjan a également la particularité d’être ceinturée par un réseau lagunaire dense. Voir fig 1

Depuis 1960, la végétation ivoirienne en occurrence celle d'Abidjan est en voie de disparition. Avec près de 16 millions d'hectares de forêts en 1960, il ne reste qu'environ 3,4 millions d'hectares de forêts en Côte d'Ivoire. Dans le but de mieux conserver son patrimoine forestier, l'État ivoirien a favorisé la création d'un réseau dense d'aires protégées qui couvre près de 10 $\%$ de son territoire. Malgré cela aujourd'hui le problème qui se pose est l'intrusion de l'homme dans ces espaces protégés tels que la forêt du Banco, ou parcs et sites. Les exploitations illicites réduisent de façon drastique les superficies au fil des années en témoignent plusieurs études. (Alexandre et al., 2019)

En general le climat à Abidjan est humide. Il est soumis au régime climatique de type équatorial avec des saisons chaudes et humides (Konan et al., 2016) ; le climat se subdivise le long de l'année comme suit :

Avril-juillet : grande saison de pluie 
- $\quad$ Juillet -septembre : petite saison sèche

- $\quad$ Septembre-novembre : petite saison de pluie

- $\quad$ Décembre-mars : grande saison sèche.

En saison humide, entre les vents du sud sont prédominants. L'air chargé en humidité est attiré au-dessus du golfe de guinée. Ce flux d'air humide remonte vers le nord et, une fois au-dessus du continent, se transforme en systèmes orageux nommés lignes de grain. Ces systèmes se déplacent d'est en ouest, provoquant la remontée de particules fines issues des feux de biomasse d'Afrique australe vers l'Afrique de l'ouest, arrosant toute la région (Remer et al., 2005)

En saison sèche avec des températures variant entre 29 et $32^{\circ} \mathrm{C}$, les vents du nord prennent l'ascendant et saturent l'atmosphère de poussières et de particules provenant de l'érosion du désert du Sahara et de la combustion de biomasse. La végétation est dominée la forêt primaire. Les précipitations sont abondantes avec plus de $1500 \mathrm{~mm}$ d'eau par an.

Abidjan est la capitale économique de la Côte d'Ivoire. Situé sur le littoral ivoirien, elle connaît une augmentation rapide de son activité économique (60\% du tissu industriel national) et une forte densité urbaine (1475 hab $/ \mathrm{km}^{2}$ ) (Sako et Beltrando, 2014). La Port Autonome d'Abidjan (PAA) a une situation géographique stratégique qui favorise un flux important de circulation automobile et ferroviaire entre Abidjan et les autres capitales et plusieurs industries.

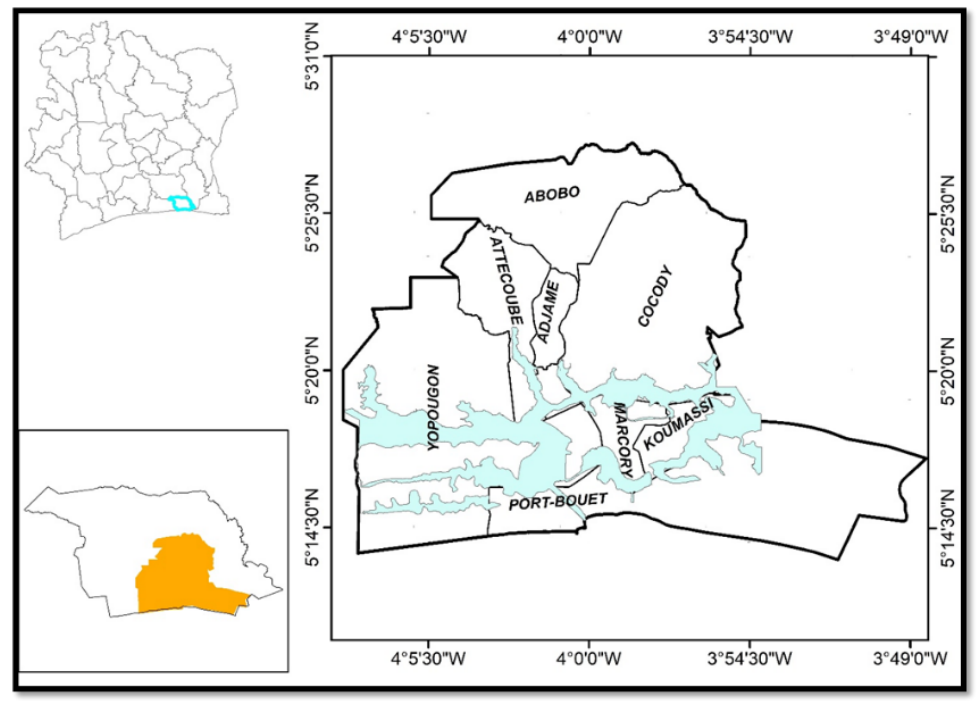

Figure 1: Carte de localisation de la ville d'Abidjan

\section{I.2. Données satellitaires}

Les images des capteurs OLI de Landsat 8 ont été essentiellement utilisées, spécifiquement des produits de niveau de prétraitement 2 de la 
collection 1 disponible les produits de réflectance de surface et de réflectance au-dessus de l'atmosphère (RS et TOA).

Les images Landsat-8 Operational Land Imager (OLI) et Thermal Infrared Sensor (TIRS) se composent de neuf bandes spectrales avec une résolution spatiale de 30 mètres pour les bandes 1 à 7 et 9 . La résolution de la bande 8 (panchromatique) est de 15 mètres. Les bandes thermiques 10 et 11 sont collectées à 100 mètres. La scène de notre zone d'étude est de chemin (path) 196 et la colonne (row) 56. Les images ont été acquises en saison sèche entre décembre et janvier. Les dates d'acquisition sont les suivantes : le 09 janvier 2015, le 28 janvier 2016, 29 décembre 2016, le 20 janvier 2019 et le 07 janvier 2020. Les années 2017 et 2018 présentaient une couverture nuageuse importante sur notre zone d'étude (voir tableau 1).

Tableau 1: Tableau recapitulatif des caractéristiques des bandes du capteur OLI de LANDSAT

\begin{tabular}{ccc}
\hline Nom de la bande & Longueur d'onde $\boldsymbol{\mu m}$ & Résolution(m) \\
\hline 1 & $0.433-0.453$ & 30 \\
2 & $0.450-0.515$ & 30 \\
3 & $0.525-0.600$ & 30 \\
4 & $0.630-0.680$ & 30 \\
5 & $0.845-0.885$ & 30 \\
6 & $1.560-1.660$ & 30 \\
7 & $2.100-2.300$ & 30 \\
8 & $0.500-0.680$ & 15 \\
9 & $1.360-1.390$ & 30 \\
10 & $10.60-11.20$ & 100 \\
11 & $11.50-12.50$ & 100 \\
\hline
\end{tabular}

\section{I.3. Calibration de la réflectance au sommet de l'atmosphère et du réflectance de surface}

En effet, les effets atmosphériques résultent de deux processus, l'absorption et la diffusion, exercées conjointement par les deux constituants majeurs de l'atmosphère, les gaz et les aérosols .Si l'on considère le rayonnement solaire comme un flux de particules élémentaires d'énergie radiative (les "photons"), l'absorption par les molécules gazeuses ou les particules d'aérosols correspond à la transformation d'une partie des photons en chaleur ; elle se traduit donc par une diminution du nombre des photons et un affaiblissement du signal mesuré dans le spectre du Soleil qui varie entre 0,2 et $4 \mu \mathrm{m}$. (Kergomard, 2000).

Les produits scientifiques Landsat de niveau 2 sont générés à partir d'algorithmes qui calculent la réflectance supérieure de l'atmosphère (TOAR), la température de luminosité du haut de l'atmosphère (TOABT), la réflectance de surface (SR) et la température de surface (ST). 
Le traitement des données de la bande de réflectance de l'imageur terrestre opérationnel (OLI) se traduit par un produit en bande estimé qui est une réflectance TOA (sans unité). Il s'agit d'une conversion des comptes numériques par un étalonnage radiométrique de niveau 1. En entrée, les données utilisées sont : la matrice elle-même, la réflectance TOA en gain et la réflectance TOA en perte (ces informations sont inscrites dans les métadonnées) et l'angle zénithal solaire. Ce calcul est effectué selon l'équation $(\mathrm{Eq}: 1)$ :

$\operatorname{Ref}(p)=(D N(p) *$ refl_gain + refl_bias $) / \cos ($ sza $)(E q: 1)$

Où

Ref(p) : Valeur de réflectance de sortie pour pixel

DN(p): Input DN value (linearized and bias corrected) par pixel 'p'.

refl_gain : Gain de réflectance, à partir de métadonnées.

refl_bias : Biais de réflectance, à partir de métadonnées

cos(sza) : Cosinus de l'angle solaire zénithal. Cette valeur est calculée par pixel à partir des valeurs des métadonnées (angle solaire de scène et géométrie de visualisation).

Les données d'entrées sont : la bande QA de niveau 1, TOA Reflectance de niveau 1, la scene centrale de l'angle zénithale, la scène centrale de l'observation de l'angle zénithale, l'altitude du terrain, l'Ozone, la vapeur. Il est supposé que la surface soit une surface lambertienne, avec une absorption minimale de l'eau atmosphérique et d'une dispersion atmosphérique minimale de Rayleigh. Le calcul de la réflectance de surface (SR) est généré à partir de l'algorithme LaSRC selon l'équation 2

$$
\rho_{\mathrm{S}}=\frac{\frac{\rho_{\mathrm{TOA}}}{\mathrm{tgOG} * \mathrm{gOO}_{3}}-\left(\rho_{\text {atm }}-\mathrm{Xro}_{\text {rayp }}\right) * \mathrm{tgwv}_{\text {half }}-\mathrm{Xro}_{\text {rayp }}}{\operatorname{tr}_{\text {atm }}{ }^{*} \operatorname{tgw}_{\text {full }} *\left(1+\mathrm{S}_{\text {atm }}{ }^{*} \rho_{\text {partial }}\right)}(\mathrm{Eq}: 2) \text { : }
$$

Où

$\rho s$ : Réflectance de surface (SR).

ppartial : Réflectance partielle de surface, décrite ci-dessous.

Ptoa: Top of Atmosphere Reflectance (TOA-R)..

tgOG :Transmission gazeux d'autres gaz..

$\operatorname{tgO}_{3}$ : Transmission gazeux de l'ozone. La variable de code est 'tgoz', souvent combinée avec d'autres gaz comme 'tgo = tgog * tgoz'. 'roatm'.

$\rho_{\mathrm{atm}}$ : Réflectance atmosphérique intrinsèque. La variable de code est

Tgwvfull : Transmission gazeux de la colonne complète de vapeur d'eau atmosphérique. La

tgwVhalf:Transmission gazeux de la moitié de la colonne de vapeur d'eau atmosphérique.

Xrorayp : Rayleigh scattering reflectance.

$\operatorname{Tr}_{\mathrm{atm}}$ : Transmission atmosphérique totale. 
$\mathrm{S}_{\mathrm{atm}}$ : Albétie sphérique atmosphérique.

. La « réflectance partielle de surface », ppartial, est une approximation de la réflectance totale de surface qui est utilisée comme aide au calcul selon l'équation 2 :

$$
\rho_{\text {partial }}=\frac{\frac{\rho_{\mathrm{TOA}}}{\operatorname{tgOG} * \mathrm{tgO}_{3}}\left(\rho_{\mathrm{atm}}-\mathrm{Xro}_{\text {rayp }}\right) * \text { tgwv }_{\text {half }}-\mathrm{Xro}_{\text {rayp }}}{\operatorname{tr}_{\text {atm }}{ }^{*} \mathrm{tgwv}_{\text {full }}} \quad(\mathrm{Eq}: 3):
$$

\section{I.4. Ajustement des valeurs des TOA et de RS et déduction de la réflectance de surface.}

En effet, les données de sortie des traitements plus haut donnent des valeurs brutes dont les comptes numériques sont en nanomètre. Il est question de faire une conversion de ces données en micromètre. Ces valeurs ont été multipliées par un coefficient multiplicateur ayant pour valeur constante de 0.0001 (Jenkerson, 2020).

$$
\mathrm{DN}(\beta)=\mathrm{R}(\beta \mathrm{x}) * 0.0001 \text { (Eq :4) }
$$

Où convertie ;

$\mathrm{DN}(\beta)$ : la bande d'image (TOA ou RS) en compte numérique numérique.

$\mathrm{R}(\beta \mathrm{x})$ : le numéro la bande d'image (TOA ou RS) en compte

La réflectance de surface (RS) déduite de la réflectance mesurée par le satellite (réflectance au sommet de l'atmosphère, TOA) permet de déterminer la réflectance atmosphérique de la bande (Remer et al., 2005) à travers l'équation suivante :

$$
\text { Ratm= TOA-RS (Eq :5) }
$$

\section{I.5. Calcul des concentrations des $\mathbf{P M}_{2.5}$}

Les estimations des polluants atmosphériques $\mathrm{PM}_{2.5}$ sont déduites de la relation qui existe entre l'épaisseur optique et les polluants en question (Glantz et al., 2007) et par ricochet entre l'épaisseur optique et la réflectance atmosphérique. Hadjimitsis, 2009 montrer une relation étroite entre les $\mathrm{PM}_{2.5}$ et l'épaisseur optique décrite par l'équation suivante :

$$
\mathrm{AOD}=\mathrm{a} * \mathrm{R}(\beta)(\mathrm{Eq}: 6)
$$

où

a est le coefficient de concentration mesurée

$\mathrm{R}(\beta)$ est le reflet atmosphérique de la longueur d'onde $(\beta)$ du satellite

Les coefficients constants de cette équation ont été obtenus de la relation linéaire entre les valeurs discrètes des $\mathrm{PM}_{2.5}$ recueillies au sol et les réflectances atmosphériques.

$\mathrm{PM}_{2.5}=\mathrm{a} \times \mathrm{R}(\beta)(\mathrm{Eq}: 7)$

Pour une meilleure précision, il est important d'étendre l'équation sur plusieurs bandes spectrales. 


$$
\mathrm{PM}_{2.5}=\mathrm{a} 0 \times \mathrm{R}(\beta 1)+\mathrm{a} 1 \times \mathrm{R}(\beta 2)+\ldots(\mathrm{Eq}: 8)
$$

Selon plusieurs sources (Hadjimitsis et al., 2009), (Mozafari et al., 2019), les bandes les mieux corrélées pour l'estimation des $\mathrm{PM}_{2.5}$ sont les bandes 1, 3 et 4, qui sont respectivement les bandes «Bleu », "Rouge » et « Proche Infrarouge ». Ainsi, les estimations des concentrations des $\mathrm{PM}_{2.5}$ ont donc été générées à l'aide de l'algorithme développé par Mozafari et al., (2019), testé donnant un coefficient de corrélation R de 0.75 et RMSE à 8.41. L’algorithme se présente de la manière suivante :

$$
\mathrm{PM}_{2.5}=369 \times \mathrm{R}(\beta 1)-197 \times \mathrm{R}(\beta 3)-376 \times \mathrm{R}(\beta \mathrm{d} 4)+3.2(\mathrm{Eq}: 9)
$$

\section{Résultats}

\section{II.1. Repartition spatiale des PM2.5 dans la ville d'Abidjan entre 2015 et 2020}

En effet, cette ligne directrice est une mesure de réduction à laquelle les pays devraient se calibrer pour éviter ou du moins réduire les risques sanitaires liées à la pollution atmosphérique.

Les estimations qualitatives de ce travail sont représentées par des plages de couleurs :

- les concentrations entre $0-25 \mu \mathrm{g} / \mathrm{m}^{3}$ sont représentées en couleur «bleu » et considérée comme des concentrations moyennes c'est-àdire acceptable

- les concentrations entre $25-35 \mu \mathrm{g} / \mathrm{m}^{3}$ sont représentées en couleur « jaune » et considérée en estimation dangereuse

- les concentrations allant de $35 \mu \mathrm{g} / \mathrm{m}^{3}$ et plus sont représentés en « rouge » et considérés comme dangereuse et alarmante

A la date du 09 Janvier 2015, la concentration moyenne est dominante sur tout l'espace Abidjanais. Les concentrations fortes sont également dans toutes les communes avec une disparité presqu'uniforme. Les concentrations très fortes sont plus localisées en des points. On les retrouve dans presque toutes les communes sauf à Attécoubé et au plateau. Notons que les concentrations fortes sont plus ou moins importantes au Nord de Yopougon, à Koumassi, à Adjamé, et disséminées à Abobo, à Cocody et Port_Bouet. Ici, les variations des concentrations sont entre 20 et $42 \mu \mathrm{g} / \mathrm{m}^{3}$.

La carte du 28 janvier 2016, présente des concentrations qui varient entre 22 et $50 \mu \mathrm{g} / \mathrm{m}^{3}$. Les fortes concentrations sont spatialement denses au niveau de la commune d'Abobo, ou elles occupent visiblement environ les trois quarts de sa superficie. Yopougon, Koumassi, Port-Bouet, Marcory, Adjamé, et Cocody présentent également des concentrations très élevées avec un étalement important dans l'espace. Les concentrations moyennes occupent les espaces restant à Cocody, à Abobo, à Yopougon, Port-Bouet et à Adjamé.

Les concentrations du 29 décembre 2016, varient entre 20 et $44 \mu \mathrm{g} / \mathrm{m}^{3}$. Les concentrations moyenne s'observent sur l'ensemble de la ville. 
Cependant, les concentrations élevées et très élevées sont relevées respectivement dans les communes d'Abobo centre et Yopougon Nord.

Sur la figure 2, les Niveaux de $\mathrm{PM}_{2.5}$ varient entre 23 et $45 \mu \mathrm{g} / \mathrm{m} 3$. Les concentrations sont très élevées à l'Ouest d'Abobo. Elles se confondent aux concentrations élevées au Nord de Yopougon, et sur toute l'étendue de Cocody. Les concentrations élevées sont dominantes su l'ensemble du territoire abidjanais. Les concentration moyennes sont discernables au Sud de la ville.

Concernant l'année 2020, on note une très forte concentration des $\mathrm{PM}_{2.5}$ dans toutes les communes à part celles de Koumassi, Treichville et une concentration mixte à Port-Bouet. Les niveaux de concentrations varient entre 23 et $43 \mu \mathrm{g} / \mathrm{m}^{3}$. Cette concentration élevée s'explique par une vague de froid et de vent passant chargée en poussière dans la période du 05 janvier 2020, tandis que l’image a été enregistrées le 07 janvier 2020.
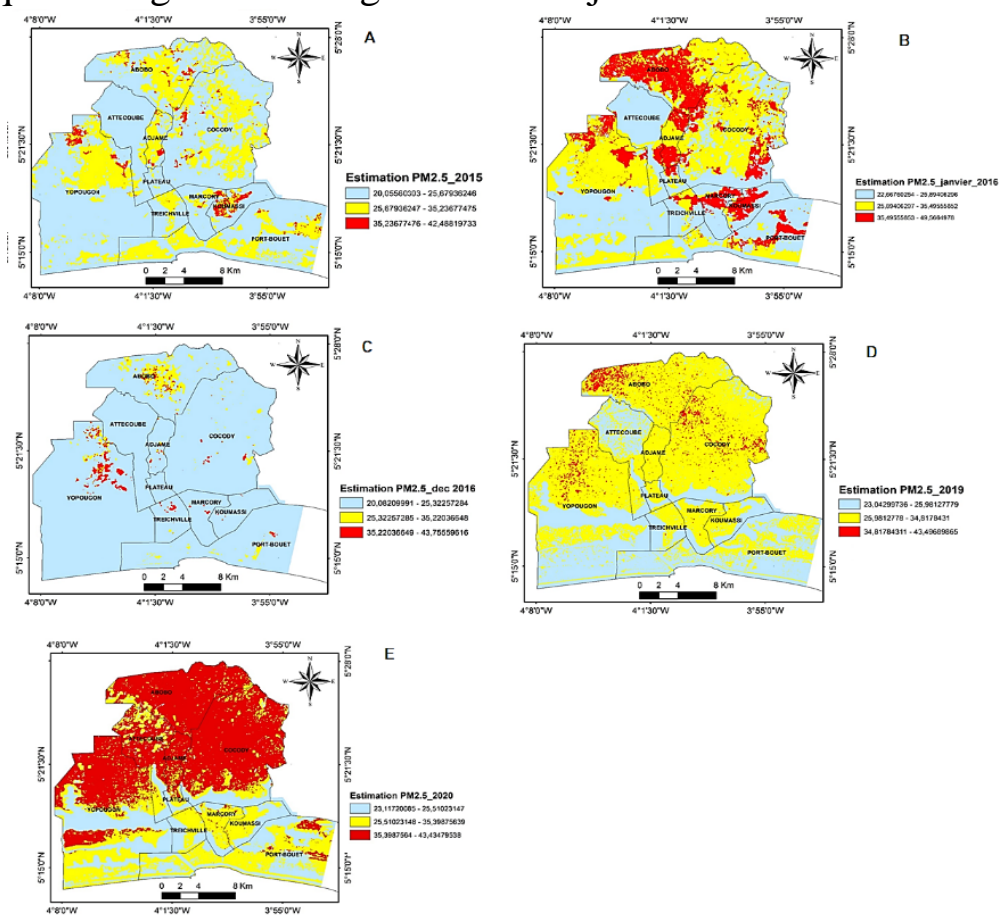

Figure 2: Cartes des pollutants atmosphériques $\mathrm{PM}_{2.5}$ entre 2015 et 2020 de la ville d'Abidjan.

\section{II.2. Evolution des concentrations $\mathbf{P M}_{2.5}$ dans la ville d'Abidjan entre 2015 et 2020}

Les concentrations journalières de $\mathrm{PM}_{2.5}$ en saison sèche de 2015 à 2020 sont des concentrations à fortes valeurs. En 2015, la concentration moyenne est de $31 \mu \mathrm{g} / \mathrm{m}^{3}$, en janvier 2016, la concentration moyenne est de $36 \mu \mathrm{g} / \mathrm{m} 3$, en décembre 2016 la concentration moyenne est de $32 \mu \mathrm{g} / \mathrm{m}^{3}$; en 
2019, la concentration moyenne est de $33 \mu \mathrm{g} / \mathrm{m}^{3}$; et en 2020, la concentration moyenne est également de $33 \mu \mathrm{g} / \mathrm{m}^{3}$.

La concentration minimale soit moyenne générale de la concentration de $\mathrm{PM}_{2.5}$ à Abidjan varie de 20 à $23 \mu \mathrm{g} / \mathrm{m}^{3}$. Cet intervalle est acceptable selon le seuil de l'OMS. Cependant les valeurs de concentration maximales varient entre 40 et $50 \mu \mathrm{g} / \mathrm{m}^{3}$. Cette variation est presque le double des concentrations du seuil OMS fixé à $25 \mu \mathrm{g} / \mathrm{m}^{3}$. Néanmoins sur toutes les années on observe une évolution plutôt constante des $\mathrm{PM}_{2.5}$ avec une légère hausse en janvier 2016 (voir fig 3).

Les communes d'Abidjan présentent donc des tendances de concentrations allant de concentrations très fortes ou très élevées à moyenne. Les communes ou les concentrations sont persistantes chaque année est : Marcory, Abobo, Port-Bouet, Cocody, Adjamé, Yopougon. Cela pourrait s'expliquer par la présence d'activité humaine intense comme le trafic routier, la présence de zone industrielles, la combustion de biomasses, comme le bois ou le fumage de poisson à certains endroits des communes. Par ailleurs, il faut également relever que les communes d'Attécoubé et du plateau sont moins impactées par la présence des $\mathrm{PM}_{2.5}$. Cela pourrait s’expliquer par la présence de végétation ou de plan d'eau qui influencent les niveaux de concentration des polluants atmosphériques.

Ainsi, dans la partie suivante, la réalisation d'une carte d'occupation du sol couplé au cartes de concentration nous permettra de corroborer ou non ces hypothèses (voir figure 3 ).

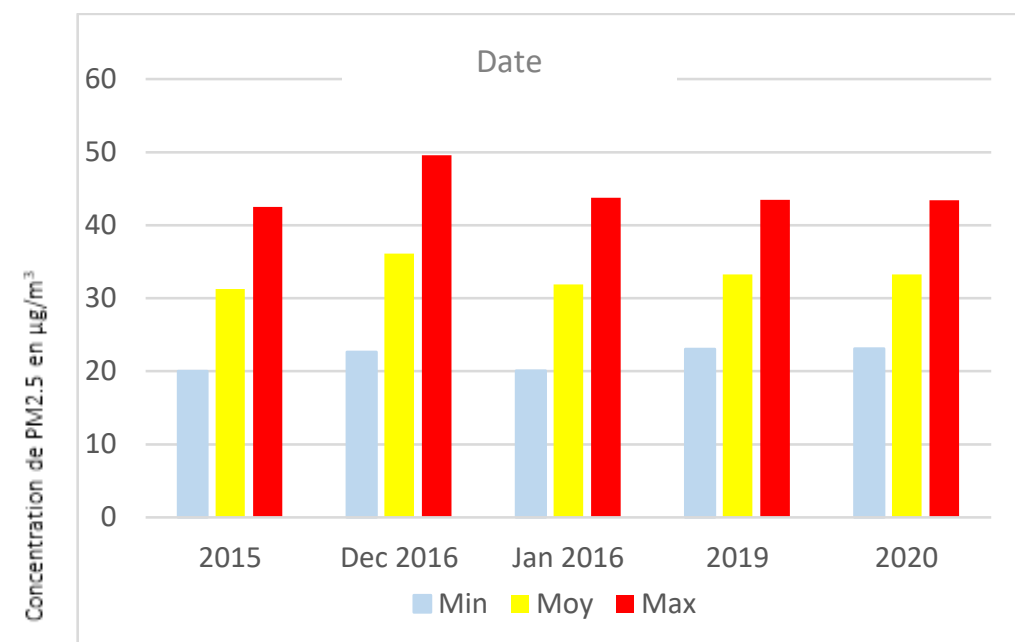

Figure3 : Evolution des concentrations des polluants atmosphériques $\mathrm{PM}_{2.5}$ entre 2015 et 2020 de la ville d’Abidjan 


\section{II.3. Discussion}

La télédétection active est l'un des meilleurs outils pour l'étude de l'estimation des aérosols de notre époque. De plus en plus performante, ce domaine est très prometteur.

En effet, en télédétection plusieurs types de capteur sont permettent de déterminer les niveaux de concentration des aérosols de $\mathrm{PM}_{2.5}$. La régression linéaire simple permet d'exprimer la concentration d'un polluant en fonction d'une seule variable (Ionescu, 2013) qui est dans notre cas la réflectance atmosphérique des bandes (elle a servie de variable unique). Dans un cas général, pour étudier les polluants atmosphériques avec le modèle de régression linéaire plusieurs variables sont utilisées : température humidité, vitesse du vent etc. On parle de régression linéaire multiple.

La donnée satellitaire utilisée pour cette étude est celle produite par le Capteur OLI de LANDSAT 8. Ce capteur a une résolution spatiale de 30*30m. Géostationnaire, il enregistre les informations sur une même zone a une résolution temporelle de 16 jours. Il offre des images de qualité sur au moins 12 bandes spectrales selon le niveau de traitement l'image. Par ailleurs, l'inconvénient est qu'au niveau de la zone tropicale les cumulus en forte densité réduisent l'exploitation de ces images.

La méthode utilisée pour cette étude à consisté à déterminer la corrélation entre l'épaisseur optique et la réflectance d'atmosphérique d'une part et entre la réflectance de surface et les concentrations de $\mathrm{PM}_{2.5}$ d'autre part .Pour chaque bande, RS a été déduite de TOA afin d'obtenir la réflectance atmosphérique. En effet, il a été démontré dans certaines une relation étroite entre le AOD et la concentration des $\mathrm{PM}_{2.5}$ (Vidot et al., 2007) également entre la réflectance de surface et l'AOD. Partant de ce fait, il a été déduit une relation étroite entre les $\mathrm{PM}_{2.5}$ et la réflectance atmosphérique. Cette déduction à permis de déterminer le coefficient de corrélation entre les données de $\mathrm{PM}_{2.5}$ et la réflectance atmosphérique (Chu et al., 2003, Sifakis et al., 2002 Chen et al., 2018).

Les particules sont des substances qui agissent directement sur la santé des populations par inhalation sur une certaine période d'exposition. Pour prévenir ces risques de santé, l'OMS en 2005 a fixé le niveau de concentration moyen de $\mathrm{PM}_{2.5}$ tolérables à $25 \mu \mathrm{g} / \mathrm{m}^{3}$ sur 24 h et $10 \mu \mathrm{g} / \mathrm{m}^{3}$ par an.

Les résultats obtenus de notre étude révèlent qu'en janvier 2015 est de $31 \mu \mathrm{g} / \mathrm{m}^{3}$, en janvier 2016 est de $36 \mu \mathrm{g} / \mathrm{m}^{3}$, en décembre $201632 \mu \mathrm{g} / \mathrm{m}^{3}$, en janvier 2019 est de $33 \mu \mathrm{g} / \mathrm{m}^{3}$ et enfin en janvier 2020 est de $33 \mu \mathrm{g} / \mathrm{m}^{3}$. Avec une estimation de concentration générale de $33 \mu \mathrm{g} / \mathrm{m}^{3}$.

Les estimations de concentration de $\mathrm{PM}_{2.5}$ en ces différentes dates sont plutôt constantent mais élevés par rapport au seuil de l'OMS. Les travaux de Gnamien et al.(2020) on relevé après l'analyse de la contribution relative des particules fines aux particules grossières que les $\mathrm{PM}_{2.5}$ contribuent aux $\mathrm{PM}_{10}$ à 
moins de $40 \%$ à Korhogo mais à plus de $50 \%$ à Abidjan. Les concentrations moyennes quotidiennes de $\mathrm{PM}_{2.5}$ sont toutes supérieures aux normes de l'OMS et sont 2 à 8 fois plus élevées à Korhogo, tandis qu'à Abidjan elles sont jusqu'à 4 fois plus élevées.

Une autre étude menée sur Abidjan sur les concentrations de $\mathrm{PM}_{2.5}$ par échantillonnage a monté que nos valeurs sont très élevées. Il estime les concentrations de $\mathrm{PM}_{2.5}$ oscillant entre 2.2 et $18,9 \mu \mathrm{g} / \mathrm{m}^{3}$ (Djossou et al., 2018).

Les résultats ont montré que la commune de Cocody était une zone de concentration très forte de $\mathrm{PM}_{2.5}$. Cette hausse de la concentration des $\mathrm{PM}_{2.5}$ à Cocody est causé le flux du trafic routier importante (Tra et Adou, 2017). La pollution automobile notamment la mise en circulation des vieilles voitures explique donc cette concentration mais aussi les mauvaises pratiques adoptées par les automobilistes.

Les résultats ont montré que la commune de Yopougon également concentre dans sa partie Nord de fortes concentrations de $\mathrm{PM}_{2.5}$. En effet, au nord de la commune est implantée une zone industrielle (chevallier, 1983) qui constitue une source de pollution en plus du trafic routier également intense. Par la vitrine d'Abidjan, les émissions anthropiques en Afrique contribuent de façon importante à la pollution atmosphérique. Selon Adon, l'activité informelle dans cette commune est dominante : le fumage de poisson par les femmes y est l'une des premières sources d'émission. Les prélèvements ont montré une concentration en $\mathrm{PM}_{2.5}$ de $133.7 \mu \mathrm{g} / \mathrm{m}^{3}$ (Adon, 2019). Ce qui dépasse largement les concentrations estimées dans cette étude. Cela s'explique sur plusieurs raisons. D'abord, nos estimations sont faites à l'échelle de la ville, tandis que lui a l'échelle de la commune. Aussi, sa méthode a consisté à faire des prélèvements directs à l'aide d'instruments de mesures.

Les résultats obtenus ont montré la presence de polluants atmospheriques le 07 janvier 2020 sur une grande partie du territoire de la ville d'Abidjan. Cette presence avait annoncée par le SODEXAM pour debut Janvier 2020.

\section{Conclusion}

Au terme de notre analyse nous retenons que la télédétection est un outil qui offre une large gamme d'application. Il est exploité dans divers domaines aussi bien pour la cartographie des entités d'occupation sol qu'à la cartographie des polluants atmosphériques. Elle a permis de faire une cartographie de la répartition spatiale des polluants à Abidjan et les différents niveaux de concentration. Les résultats obtenus après le traitement des données ont montré une tendance évolutive des $\mathrm{PM}_{2.5}$ dans la ville d'Abidjan. Ces tendances sont pour la plupart au-dessus du seuil de la qualité de l'air 
décrite par l'OMS. Ainsi a l'issue de cette étude, il est important d'alerter les populations sur leur niveau d'exposition afin qu'elle à adoptent des attitudes contribuant à réduire l'emission des particules polluantes.

\section{References :}

1. Adon, A.J. (2019). Évaluation de l'impact sur la santé de l'aérosol de combustion pour différentes sources urbaines en Afrique de l'Ouest en saison sèche et humide: caractérisation physico-chimique et toxicologique. Université Toulouse III - Paul Sabatier, Toulouse.

2. Alexandre Y. K., Marcel Y. B., Yves A. Y. C. et Célestin A. Y. (2019). Enjeux des Activités Humaines dans le Maintien de la Diversité Végétale des Forêts Marécageuses de la Sous-Préfecture de GrandLahou sur le Littoral Ivoirien. European Scientific Journal ESJ, vol. 15, n 15 , pp. 24.

3. Aoun Y. (2017). Les aérosols atmosphériques, qu'est-ce que c'est ? HAL Id: hal-01555621, pp. 20.

4. Basly L. (2000). Télédétection pour la qualité de l'air en milieu urbain. Thèse de doctorat, sciences de technologies de l'information et de la communication, Université de Nice Sophia Antipolis, France. $182 \mathrm{p}$.

5. Basly L. (2000). Télédétection pour la qualité de l’air en milieu urbain. $\mathrm{PhD}$ Thesis,

6. Boucher O. (2012). Les aérosols atmosphériques, in : O. Boucher (Éd.), Aérosols atmosphériques : Propriétés et impacts climatiques. Ingénierie et développement durable. Springer, Paris, pp.7-16

7. Cachon F. B. A. (2013). Étude de pollution atmosphérique en Afrique Sub-Saharienne : Cas de Cotonou (Bénin): Caractérisation physicochimique des matières particulaires d'origine urbaine et impact toxicologique sur des cellules épithéliales bronchiques humaines (BEAS-2B) cultivées in vitro., Université du Littoral Côte d'Opale; Université d'Abomey-Calavi (Bénin), Benin, 322 p. Consulté à l'adresse https://tel.archives-ouvertes.fr/tel-01367378

8. Cerema V. B. (2014). Détection de particules fines par LIDAR. Bron Cedex Consulté à l'adresse www.cerema.fr

9. Chen Y., Luo X.-S., Zhao Z., Chen Q., Wu D., Sun X., Wu L. et Jin L. (2018). Summer-winter differences of PM2. 5 toxicity to human alveolar epithelial cells (A549) and the roles of transition metals.Elsevier Ecotoxicology and environmental safety, vol. 165, pp. 505-509.

10. Chevallier P. (1983). YOPOUGON (COTE D'IVOIRE).

11. Chu Da, YJ Kaufman, G. Zibordi , JD Chern , J. Mao , C. Li , BN Holben Surveillance globale de la pollution de l'air au-dessus des 
terres d'EOS-Terra MODIS J. Géophys. Rés. , 108 ( D21 ) ( 2003 ), p. 4661.

12. Djossou, J., Léon, J.F., Akpo, A.B., Liousse, C., Yoboué, V., Bedou, M., Bodjrenou, M., Chiron, C., Galy-Lacaux, C., Gardrat, E., Abbey, M., Keita, S., Bahino, J., Touré N’Datchoh, E., Ossohou, M., Awanou, C.N. (2018). Mass concentration, optical depth and carbon composition of particulate matter in the major southern West African cities of Cotonou (Benin) and Abidjan (Côte d'Ivoire). Atmospheric Chemistry and Physics 18, 6275-6291.

13. Hadjimitsis D. G., Clayton C. R. I. et Retalis A. (2009). The use of selected pseudo-invariant targets for the application of atmospheric correction in multi-temporal studies using satellite remotely sensed imagery. International Journal of Applied Earth Observation and Geoinformation, vol. 11, $n^{\circ}$ 3, pp. 192-200.

14. Horvath J. S. (1997). The compressible inclusion function of EPS geofoam.Elsevier Geotextiles and Geomembranes, vol. 15, n 1-3, pp. 77-120.

15. Ionescu A., Mayer E., Colda I. (1996). Méthodes mathématiques pour estimer le champ de concentration d'une polluant gazeux à partir des valeurs mesurées aux points dispersés. Pollution Atmosphérique, janvier-mars 1966, pp 78-89.

16. Jenkerson C. (2020). Landsat 8 Collection 1 (C1) Land Surface Reflectance Code (LaSRC) Product Guide., vol. Version 3.0, pp. 38.

17. Kergomard C. (2000). Pratique des corrections atmosphériques en télédétection : utilisation du logiciel 5S-PC. CNRS-UMR Géographiecités 8504 Cybergeo :European Journal of Geography.

18. Kouadio K., Adje J., Maesano C., Veronique Y. et Liousse C. (2019). PM2. 5 concentration and Health impact assessment in Abidjan hospital, Côte d'Ivoire.LWW Environmental Epidemiology, vol. 3, pp. 215.

19. Mozafari S. M., Hasanlou M. et Arefi H. (2019). Air pollution estimation using aerosol optical thickness by oli images in tehran. ISPRS - International Archives of the Photogrammetry, Remote Sensing and Spatial Information Sciences, vol. XLII-4/W18, pp. 779-782.

20. Organization W. H. (2016). Ambient air pollution: A global assessment of exposure and burden of disease. World Health Organization

21. Péré, J.-C., V. Pont, M. Mallet et B. Bessagnet. 2009. Cartographie des concentrations de surface de PM10 dérivées d'observations satellitaires de l'épaisseur optique des aérosols au-dessus du sud-est de la France. Recherche atmosphérique 91 (1) : 1 - 8. 
22. Remer L. A., Kaufman Y. J., Tanré D., Mattoo S., Chu D. A., Martins J. V., Li R.-R., Ichoku C., Levy R. C. et Kleidman R. G. (2005). The MODIS aerosol algorithm, products, and validation. Journal of the atmospheric sciences, vol. 62, $\mathrm{n}^{\circ}$ 4, pp. 947-973.

23. Sako N. et Beltrando G. (2014). Dynamiques spatiales récentes du Parc National du Banco (PNB) et stratégies de gestion communautaire durable de ses ressources forestières (District d'Abidjan en Côte d'Ivoire). Pôle de recherche pour l'organisation et la diffusion de l'information géographique (CNRS UMR 8586) EchoGéo, nº 30.

24. Sifakis I.S. (1992). Potentialités de l'imagerie à haute résolution spatiale pour le suivi de la repartition de pollutions atmosphériques dans la basse troposphère : Etude du cas d'Athènes. Thèse de Doctorat, Chimie de la Pollution Atmosphérique et Physique de l'Environnement, Université Paris 7 Denis Diderot, Paris, 292 p.

25. Stromatas S. (2013). Contributions relatives des aérosols minéraux et de feux sur la qualité de l'air en Euro-Méditerrannée. $\mathrm{PhD}$ Thesis,

26. Techarat P., Veawab A., Piwowar J. M. et Rahman M. (2018). Mapping Spatial Distribution of Ambient Particulate Matter and Sulfur Dioxide Concentrations Using LANDSAT Data : A Case Study for the Province of Saskatchewan, Canada. Advances in Environmental Research, vol. 62.

27. Tra F, Adou Yedlock Marie Christiane. (2017), Transport Routier Et Pollution De L'air : Etude De La Conscience Environnementale Des Automobilistes Dans La Commune De Cocody (Abidjan), Vol 13 No 8 (2017): édition de mars de l'ES.

28. Vidot, J, Richard S, et Didier R (2007). Atmospheric Particulate Matter (PM) Estimation from SeaWiFS Imagery . Remote Sensing of Environment 111, $\mathrm{n}^{0} 1$ (novembre 2007) 1-10. 